
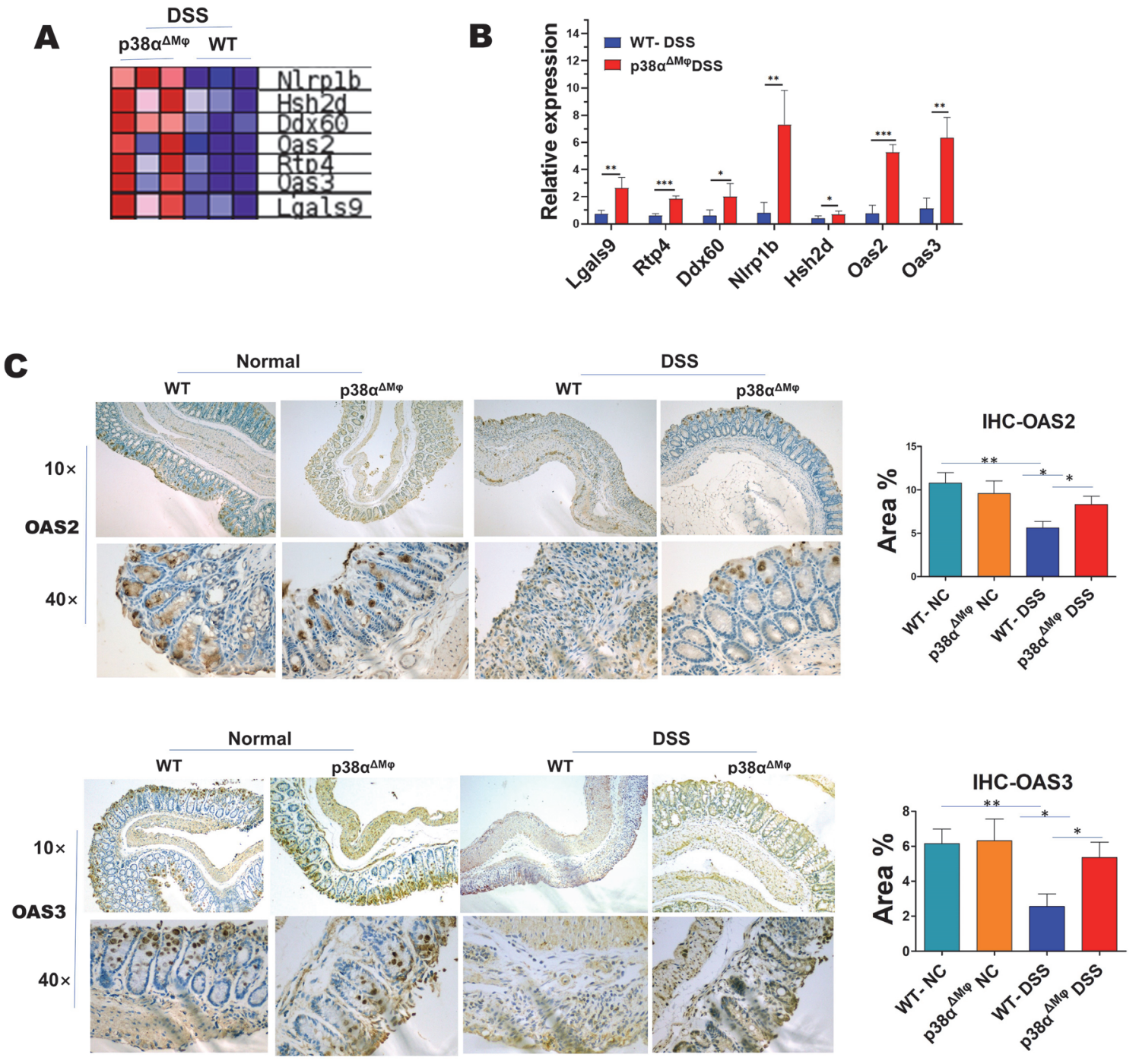

Abstract IDDF2021-ABS-0097 Figure 7

\section{IDDF2021-ABS-0098 COMPARISON OF CURATIVE EFFECT BETWEEN METFORMIN AND MESALAZINE IN THE TREATMENT OF EXPERIMENTAL COLITIS}

${ }^{1}$ Jun Deng* ${ }^{2}$ Lishan Zeng, ${ }^{3}$ Le Liu, ${ }^{3}$ Ye Chen. ${ }^{1}$ The Sixth Affiliated Hospital, Sun Yat-sen University, China; ${ }^{2}$ Guangzhou Panyu Central Hospital, China; ${ }^{3}$ Nanfang Hospital, Southern Medical University, China

\subsection{6/gutjnl-2021-IDDF.35}

Background To evaluate the different efficacy in the treatment of mice colitis between metformin and mesalazine.
Methods C57BL/6 male mice aged 6-8 weeks were given ad libitum access to $3 \%$ dextran sulphate sodium to induce colitis. The treatment groups were administered with metformin and mesalazine, respectively. The body weight, colon length, disease activity index(DAI), histopathological changes and the expression of pro-inflammatory cytokines in each group were evaluated.

Results Compared to the DSS group, mice treated with metformin or mesalazine simultaneously experienced significantly less bodyweight loss, less DAI scores (IDDF2021ABS-0098 Figure 1) and less colon length shortening (IDDF2021-ABS-0098 Figure 2). Histological examination
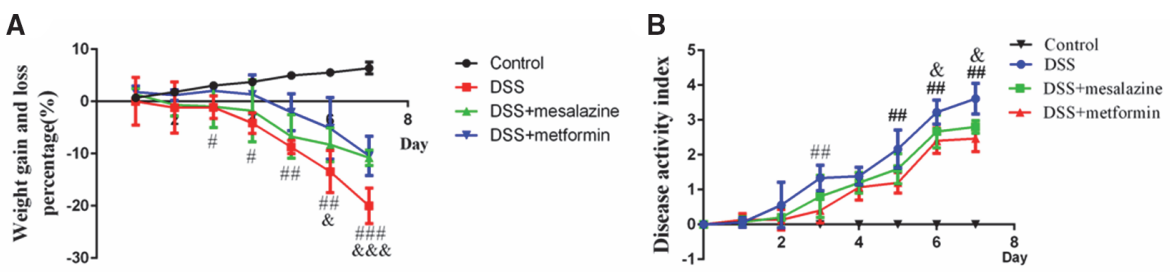

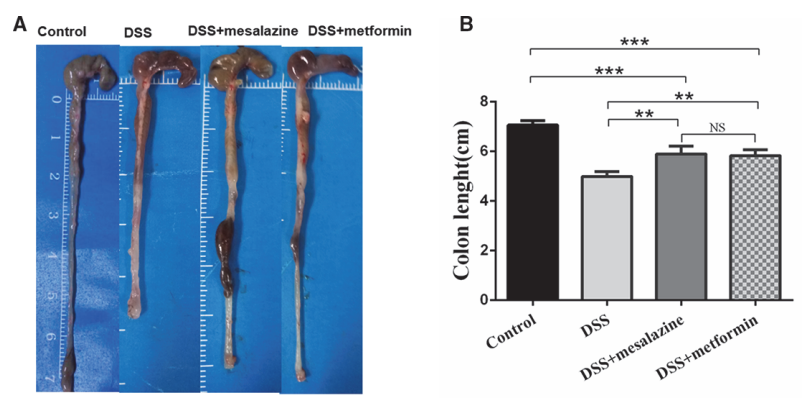

showed better histological scores in mesalazine treatment group and metformin treatment group (IDDF2021-ABS0098 Figure 3). Moreover, metformin or mesalazine could significantly inhibit the mRNA expression levels of IL-6, TNF- $\alpha$ and IL-1 $\beta$ (IDDF2021-ABS-0098 Figure 4). But there was no significant difference between the two treatment groups.

Conclusions Metformin can ameliorate DSS-induced experimental colitis in mice, and its curative effect is not inferior to that of mesalazine.

Abstract IDDF2021-ABS-0098 Figure 2
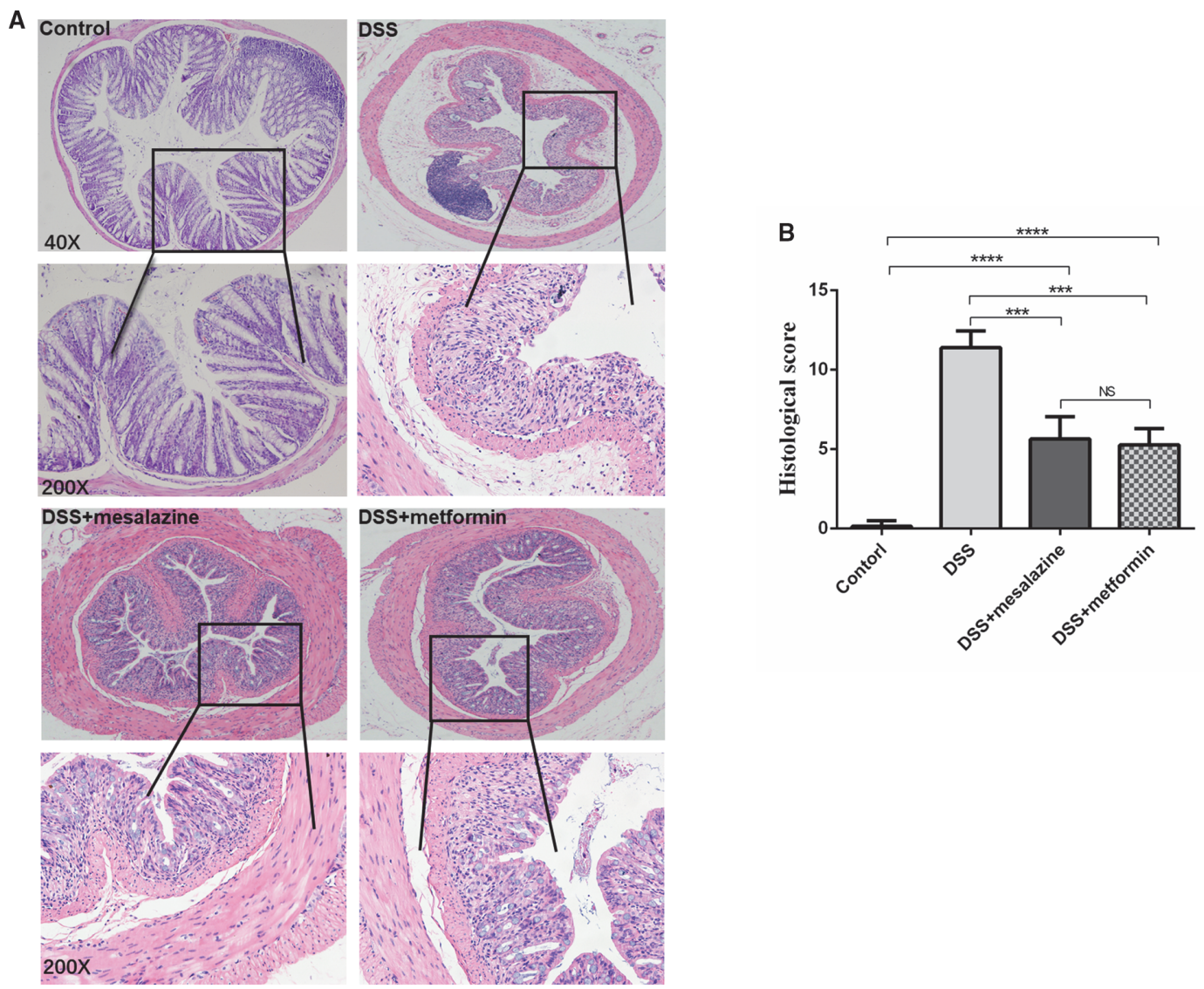

Abstract IDDF2021-ABS-0098 Figure 3

A

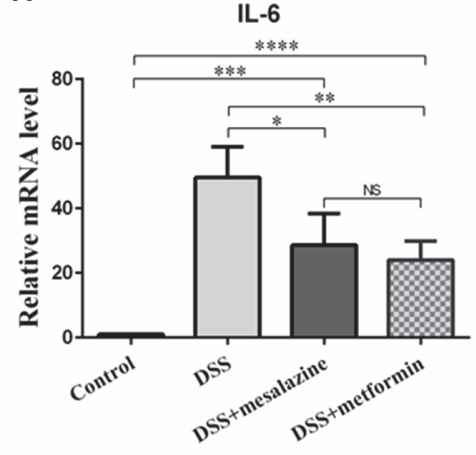

B

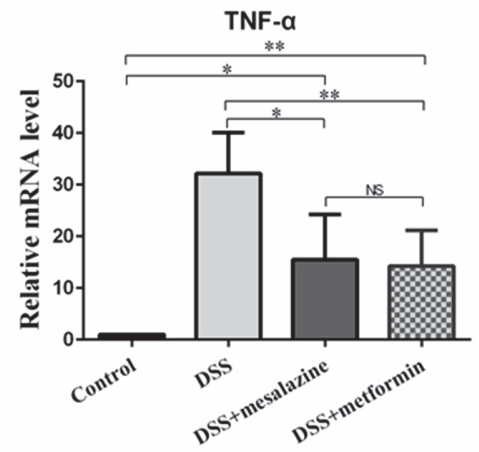

C

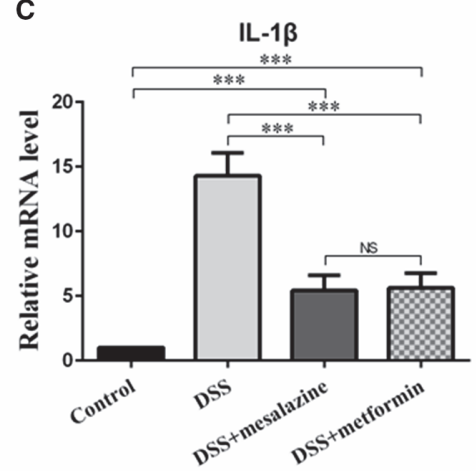

